Volume 3

Issue 4 -- Cardiovascular Aging

Article 16

$11-11-2016$

\title{
Quality Improvement of Procedural Services in Family Medicine Residency Clinics
}

Keisha Rogers

Nora Guschwan

Lisa Sullivan Vedder

Follow this and additional works at: https://aah.org/jpcrr

Part of the Family Medicine Commons, and the Interprofessional Education Commons

\section{Recommended Citation}

Rogers K, Guschwan N, Sullivan Vedder L. Quality improvement of procedural services in family medicine residency clinics. J Patient Cent Res Rev. 2016;3:238-9.

Published quarterly by Midwest-based health system Advocate Aurora Health and indexed in PubMed Central, the Journal of Patient-Centered Research and Reviews (JPCRR) is an open access, peer-reviewed medical journal focused on disseminating scholarly works devoted to improving patient-centered care practices, health outcomes, and the patient experience. 
used, including variants of delirium, altered mental status, acute confusional state, acute brain syndrome, acute brain failure, metabolic encephalopathy, predict, predictive, prediction, models, modeling, scores, scoring, tests, testing, rules, index and indices. The bibliographies of included studies were examined, and no additional articles were referenced.

Results: To appropriately extract data from the 12 studies meeting inclusion criteria, the following parameters were used: study description, study population, delirium assessment method, incidence of delirium, and risk factors for delirium. Quality for cohort studies was assessed using "NewcastleOttawa Quality Assessment Scale" ranging from 1 to 9 (1 = poor quality, $9=$ high quality). Overall incidence of delirium in the studies ranged from $4 \%$ to $26 \%$. Most common risk factors for delirium were dementia, decreased functional status, blood urea nitrogen to creatinine ratio, infection and severe illness. Other variables less common were alcohol, malignancy, history of delirium, older age, medications, physical restraints, malnutrition, admitted from other than home and an iatrogenic event. The quality of studies ranged from 4 to 8 .

Conclusion: This systematic review summarizes the medical literature on risk prediction models for delirium in hospitalized older patients. We will use this information to develop an EHRgenerated delirium risk prediction model to be used by the "Hospital Elder Life Program" to reduce delirium incidence.

\section{Mind and Body Training to Improve Functioning and Coping With Chronic Pain: A Pilot Study}

\author{
Olga Valieva, Leah M. Welsh, Betty Amuzu, Niraj \\ Nijhawan, Jessica J.F. Kram
}

\section{Department of Obstetrics and Gynecology, Aurora Sinai Medical Center; Department of Family Medicine, Aurora Health Care; Department of Obstetrics and Gynecology, Aurora UW Medical Group; Department of Anesthesiology and Obstetrics Anesthesiology, Aurora Sinai Medical Center; Center for Urban Population Health}

Background: Patients with chronic pain are often crippled by psychological distress, depression and fear. These patients also can develop altered pain perception, with enhanced brain activity in pain-responsive regions and those associated with anxiety/ depression. Exercise and meditation can impact pain-reducing brain areas and positively influence pain characteristics.

Purpose: To alter pain center activity by reducing the activation of the higher brain and deactivation of the lower brain with somatocognitive and meditative practices, with secondary aim of reducing anxiety/depression and improve overall quality of life.

Methods: We conducted a pilot study on mentally competent adult women with stable chronic pain who were resistant to conventional therapies. Our intervention consisted of an initial 8-hour session at which baseline assessments were completed with introduction to mind/body tools (i.e. deep meditation, breath work, etc.). Baseline assessments also included selfassessment using pain rating surveys, the Zung self-rating anxiety and depression scales, the World Health Organization
Quality of Life-BREF instrument, and the Conner-Davidson Resilience Scale. Following the initial session, 1.5-hour-long meetings were held weekly for 8 weeks, followed by biweekly meetings for 8 weeks, then monthly. Mind/body tools were systematically taught and reinforced during meetings. Patients kept a journal detailing their practice. Pain rating surveys were filled out monthly. All other measures were filled out at 3 and 6 months.

Results: Participating women $(\mathrm{N}=5)$ had mean age of 43.2 years and mean body mass index of $35.8 \mathrm{~kg} / \mathrm{m}^{2}$. Mean longacting narcotic (LAN) was 260, 221.6 and $248.2 \mathrm{mg}$ /day at baseline, 3- and 6-month assessments, respectively. Patients did not significantly decrease use of LAN. Additionally, no statistical difference was identified in a patient's time in pain or pain right now, resilience, anxiety and depression. However, overall quality of life improved significantly at 6-month followup (50.0 vs $25.0, \mathrm{P}=0.016)$. Following 6-month assessment, patients were highly satisfied with their experience. All (100\%) strongly agreed that the instructors responded well to questions and established good relationships with participants.

Conclusion: Intervention resulted in statistically nonsignificant decreased LAN use and reduced anxiety and depression scales, as well as statistically significant improvement in overall quality of life. Data from these patients will continue to be collected at 6-month intervals to see if there are lasting effects or further improvements.

\section{Quality Improvement of Procedural Services in Family Medicine Residency Clinics}

\section{Keisha Rogers, Nora Guschwan, Lisa Sullivan Vedder \\ Department of Family Medicine, Aurora Health Care; Department of Family Medicine, Aurora UW Medical Group}

Background: Performing common procedures in our family medicine residency clinics is often a difficult and inefficient process. A 2008 Society of Teachers of Family Medicine consensus statement on procedural training found higher job satisfaction and better financial compensation for family practitioners who performed procedures. Patient satisfaction is likely increased when minor procedures are able to be performed by their primary clinician. This would suggest a disconnect between the known benefits of providing procedural services and the ability of our residency clinics to provide those services in an efficient manner.

Purpose: To assess clinician and staff comfort with performance of common family medicine procedures and implement an intervention to improve the efficiency of procedure performance in the clinic setting.

Methods: Phase 1: Preintervention survey was distributed to physicians, residents and staff at Aurora Health Care's family medicine residency clinics. Survey evaluated comfort level of providers in performing common procedures and identifying proper equipment needed to perform procedures. Data was compiled in Microsoft Excel; statistical analysis was performed using ordinal logistic and binary regression. Phase 
2: Instructional procedures manual was created for 15 common procedures and provided to clinics. Phase 3: Postintervention survey was distributed after providers had access to procedures manual.

Results: Presurvey data revealed the majority of respondents felt neutral about their ability to perform procedures. However, residents felt more comfortable performing procedures than faculty. Postsurvey data revealed that a minority of respondents were aware of or used the procedures manual.

Conclusion: Our study demonstrated that a targeted intervention to improve the efficiency of procedure performance in the clinic setting was not well utilized. Therefore, a definitive conclusion about the ability of the intervention to improve the efficiency of procedure performance was not able to be made. Future areas of focus include better promotion of and ease of access to any selected intervention to improve efficiency of procedure performance.

\section{Prevalence of Prescription Opioid Abuse in Patients With Pain}

Samuel Godana, Ankoor Biswas, Nebiyu Biru, Abraham Getenet, Biana Leybishkis

\section{Department of Internal Medicine, Aurora Sinai Medical Center; Department of Internal Medicine, Aurora UW Medical Group}

Background: Studies showed that $20 \%$ to $30 \%$ of opioid analgesic drugs prescribed for chronic pain in the United States are misused, while the rate of opioid addiction is approximately $10 \%$. The study describes methods to identify high-risk behavior and forward recommendations to decrease opioid abuse.

Purpose: To assess prevalence and correlate multiple variables with opioid abuse and to forward appropriate recommendation to decrease the prevalence of opioid abuse in the primary care setting.

Methods: A prospective study was conducted at a primary health care clinic, Aurora Sinai Medical Center (Milwaukee, WI). Over 6 months, a total of 49 consecutive patients who take opioids were included in the study. Screener and Opioid Assessment for Patients with Pain-Revised (SOAPP-R), with a cutoff score of 18 (out of 24), is used to screen abuse/aberrant drug use. Additional variables were obtained by patient chart review. Data analysis was done using Minitab analysis software. Results: A total of 49 patients (23 males, 26 females) were included in the study. Mean age of patients was $45.7 \pm 7$ years; 22 (44.9\%) patients were on hydrocodone, $16(32.7 \%)$ on oxycodone, $7(14.3 \%)$ on tramadol, $3(6.1 \%)$ on fentanyl patch and $1(2 \%)$ on morphine and methadone. This means 33 $(66.3 \%)$ patients were on an opioid other than oxycodone. Of the 16 on oxycodone, 12 were male ( $52 \%$ of gender population) compared to 4 female ( $15.4 \%$ of gender population), $\mathrm{P}=0.013$. Five $(10.2 \%)$ patients had high SOAPP-R scores; 3 (13\%) of the males had a high SOAPP-R score compared to $2(7.7 \%)$ females. Regarding males on oxycodone, 3/12 (25\%) had high SOAPP-R scores; $1 / 4$ (25\%) females on oxycodone had a high SOAPP-R score. As age increased, the number of male patients using oxycodone trended upward. Older males on oxycodone had a higher SOAPP-R score compared to younger patients. No patient had prescriptions by more than one prescriber; 45 $(91.9 \%)$ patients had no pain contract. Urine drug screen was done in $11(22.5 \%)$ patients.

Conclusion: Prescription opioid drug abuse is not uncommon in primary care clinics. Use of the SOAPP-R, along with other appropriate patient evaluations, can help primary care providers identify high-risk behavior and decrease opioid abuse.

\section{An Interdisciplinary Process Change: Conversion of PICC Line Capping Solution From Heparin to Normal Saline}

Angela Colella, Brandon Bodager, Frank Spexarth, Natalie McDonough, Deb Kastenholz, Paul Grebe

Departments of Pharmacy, Radiology and Nursing, Aurora St. Luke's Medical Center, Aurora Health Care; AMG Imaging Services

Background: Heparin-induced thrombocytopenia (HIT) and HIT with thrombosis syndrome (HITTS) are serious conditions. Patients are at increased risk for developing HIT/HITTS with any exposure to heparin, even intravenous line flushes. Patients may be exposed to heparin multiple times each day when they have a peripherally inserted central catheter (PICC) that is flushed and capped with heparin. At Aurora Health Care, heparin is the standard capping solution for PICCs, but with a recent switch to positive pressurized caps, normal saline may be a capping option that reduces patient exposure to heparin.

Purpose: To reduce heparin exposure at a single hospital by replacing heparin with normal saline $(0.9 \%$ sodium chloride) as the standard PICC capping solution.

Methods: We implemented an interdisciplinary pilot process change and evaluated whether normal saline was noninferior to heparin for maintaining PICC patency. Primary outcome measurements of patency include alteplase use due to occlusion. Secondary objectives were any change in invasive line infection rates and cost comparisons. The baseline patency rate for the hospital was derived from data extracted through a retrospective chart review from October 2013 through October 2014.

Results: Our baseline patency rate was $65.42 \%(\mathrm{~N}=3,095)$; $33 \%$ of all PICC lines placed during the 12-month period were treated with alteplase. Patency rates during our pilot period were based on retrospective chart reviews of patients with PICC lines placed from February to May 2015. During this period, a total of 979 PICC lines were evaluated. Of these, $30.4 \%$ were treated with alteplase. This resulted in a pilot patency rate of $69.5 \%$, which is $4.1 \%$ greater than our baseline patency rate. Given the prespecified noninferiority margin of $5 \%$, our pilot demonstrates that normal saline is noninferior to heparin for maintaining PICC line patency when used with positive pressurized caps $(\alpha<0.05)$. Although number of infections increased $16 \%$, only $9 \%$ with line infections were part of our pilot. The estimated yearly cost of both capping solutions is equal, at approximately $\$ 3,960$ per our baseline 